\title{
COMO SÃO TRATADAS AS FRATURAS DIAFISÁRIAS FECHADAS DO FÊMUR NO BRASIL? ESTUDO TRANSVERSAL.
}

\author{
HOW ARE CLOSED FEMORAL DIAPHYSEAL FRACTURES TREATED IN BRAZIL? A CROSS-SECTIONAL STUDY
}

\author{
Robinson Esteves Santos Pires ${ }^{1}$, Hélıo Jorge Alvachian Fernandes², João Carlos Bellotº ${ }^{3}$. Daniel Balbachevsky', \\ FLÁVIO FalopPA ${ }^{5}$, FeRnando BaLdy dos REIS ${ }^{6}$
}

\section{RESUMO}

Realizou-se um estudo transversal no $36^{\circ}$ Congresso Brasileiro de Ortopedia e Traumatologia, onde foi pesquisada a opinião de ortopedistas brasileiros sobre aspectos do tratamento de fraturas diafisárias do fêmur no adulto. Quinhentos e sete questionários foram respondidos integralmente e encontrou-se concordância entre os ortopedistas em relação aos seguintes aspectos: configuração do traço de fratura e lesão de partes moles ou de estruturas neurovasculares como parâmetros principais para decisão sobre o tratamento; classificação das fraturas, em que a AO foi a mais adotada; haste intramedular anterógrada bloqueada fresada para tratamento das fraturas transversas e oblíquas curtas no istmo; placa ponte para o tratamento das fraturas com traço complexo; tração esquelética pré-operatória; infecção como complicação mais freqüente e uso de heparina de baixo peso molecular no pós-operatório. Houve conflito de opiniões nas seguintes questões: uso de mesa de tração para realização de osteossíntese intramedular; intervalo de tempo entre o trauma e a cirurgia; tempo de utilização de antibióticos e tempo médio de hospitalização.

Em relação à literatura, houve concordância em relação aos parâmetros principais para decisão sobre o tratamento; método de fixação das fraturas com traço simples no istmo; classificação adotada; profilaxia antitrombótica. Diferiram da literatura questões como o método de fixação das fraturas com traço complexo; tempo de utilização dos antibióticos, intervalo médio entre o trauma e a osteossíntese e tempo de internação.

Descritores: Fraturas do fêmur; Estudos transversais; Fraturas.

\section{INTRODUÇÃO}

O tratamento das fraturas diafisárias do fêmur no adulto é eminentemente cirúrgico ${ }^{(1,2,3,4,5)}$.

Existem diferentes técnicas e diversos tipos de implantes que podem ser utilizados para a estabilização destas fraturas. No entanto, os recursos hospitalares como o intensificador de ima-

\section{SUMMARY}

A cross-sectional study was performed during the $36^{\text {th }}$ Brazilian Congress of Orthopaedics and Traumatology, where the opinions of Brazilian orthopaedic surgeons addressing the treatment of femoral diaphyseal fractures in adults were surveyed. Five hundred and seven questionnaires were fully completed and the results show agreement in the following topics: fracture trace configuration and injuries of soft parts or neurovascular structures as key parameters for determining treatment; fractures classification, in which $A O$ was most frequently adopted; milled blocked anterograde intramedullary nail for treating cross-sectioned and short oblique factures at the isthmus; bridge plate for treating complex trace fractures; pre-operative skeletal traction; infection as the most frequent complication, and; postoperative low molecular weight heparin. There were opinion conflicts for the following topics: use of traction table for performing intramedullary osteosynthesis, time interval between trauma and surgery; time of antibiotics use, and; mean hospitalization time.

Regarding literature, there was agreement concerning key parameters for determining treatment; fixation method for simple-traces fractures at the isthmus; adopted classification; antithrombotic prophylaxis. Issues such as fixation method for complex-traced fractures; time of antibiotics use; average interval between trauma and osteosynthesis, and; hospitalization time were different from literature.

Keywords: Femoral fractures; Cross-sectional studies; Fractures.

gem, a disponibilidade de material adequado para a realização da osteossíntese e o treinamento da equipe cirúrgica podem influenciar na escolha do tipo de osteossíntese a ser realizada. O objetivo deste estudo é verificar como os ortopedistas brasileiros tratam os pacientes com fratura diafisária do fêmur em relação aos seguintes aspectos: parâmetros principais para decisão sobre o tipo de tratamento; classificação; opções de estabilização; utilização de

Trabalho realizado no Departamento de Ortopedia e Traumatologia da Universidade Federal de São Paulo (EPM).

Endereço para correspondência: Rua Borges Lagoa, 783 - 50 andar - Vila Clementino - São Paulo /SP - CEP 04038-032 - E-mail:robinsonesteves@ig.com.br

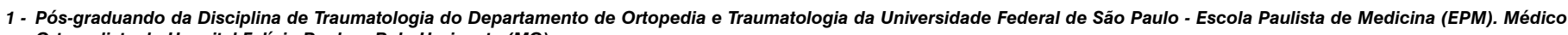
Ortopedista do Hospital Felício Rocho - Belo Horizonte (MG).

2 - Chefe do Grupo de Trauma Ortopédico, Doutor em Ciências pelo Departamento de Ortopedia e Traumatologia da Universidade Federal de São Paulo (EPM).

3 - Doutor em Ciências pelo Departamento de Ortopedia e Traumatologia da Universidade Federal de São Paulo (EPM).

4 - Pós-graduando da Disciplina de Traumatologia do Departamento de Ortopedia e Traumatologia da Universidade Federal de São Paulo (EPM).

5 - Professor Titular e Chefe do Departamento de Ortopedia e Traumatologia da Universidade Federal de Sáo Paulo (EPM).

6 - Professor Livre Docente da Disciplina de Traumatologia do Departamento de Ortopedia e Traumatologia da Universidade Federal de São Paulo (EPM).

Trabalho recebido em: 26/01/06 aprovado em 27/03/06 
mesa de tração; tipo de tração pré-operatória; intervalo médio entre o trauma e a cirurgia; tempo de internação; uso de antibióticos; complicações mais freqüentes e profilaxia antitrombótica.

\section{MATERIAL E MÉTODO}

Quinhentos e dezoito congressistas, médicos ortopedistas e residentes em ortopedia e traumatologia responderam a um questionário contendo questões referentes ao tratamento das fraturas diafisárias do fêmur durante o $36^{\circ}$ Congresso Brasileiro de Ortopedia e Traumatologia. Foram excluídos 11 questionários: oito estavam incompletos, um foi preenchido por fisioterapeuta, um por ortopedista europeu e um por clínico geral.

O questionário constava de 11 perguntas objetivas em forma de testes, englobando os seguintes aspectos: identificação (titularidade); região do país, parâmetros principais para decisão sobre o tratamento; classificação; opções de tratamento para as fraturas transversas com traço simples e para aquelas com traço complexo; utilização de mesa de tração; tração pré-operatória; intervalo de tempo entre o trauma e a cirurgia; tempo de hospitalização; uso de antibióticos; complicações mais freqüentes e profilaxia anti-trombótica. Em algumas questões, admitia-se mais de uma resposta.

Este estudo foi aprovado pelo Comitê de Ética em Pesquisa de nossa instituição. Houve adesão voluntária dos participantes. As respostas foram mantidas em sigilo, analisadas com auxílio de um software e computadas de acordo com parâmetros estatísticos.

\section{MÉTODO ESTATÍSTICO}

A presença de associação entre a escolha de tratamento preconizado nos diversos aspectos do tratamento das fraturas e algumas características dos entrevistados foi avaliada pelo teste do Qui-quadrado ou pelo teste exato de Fisher (quando aplicável). Adotou-se o nível de significância de 0,05 (=5\%), e níveis descritivos (p) inferiores a esse valor foram considerados estatisticamente significantes e representados por *.

Definiu-se que a porcentagem de respostas superior a 50\% e estatisticamente superior às demais opções, em cada aspecto, será considerada como consenso.

\section{RESULTADOS}

\section{Características dos participan-} tes: Foram respondidos 308 (61\%) questionários por ortopedistas titulares da Sociedade Brasileira de Ortopedia e Traumatologia (SBOT), $70(14 \%)$ por membros associados à SBOT e 129(25\%) por residentes em ortopedia e traumatologia.

Dos ortopedistas que responderam à pergunta sobre subespecialidade, $22 \%$ eram especialistas em quadril, 11\% em trauma, 11\% em mão, 11\% em traumatologia do esporte, $10 \%$ em joelho, $7 \%$ em coluna, $7 \%$ em pé e tornozelo, $7 \%$ em tumores músculo-esqueléticos, 5\% em ortopedia pediátrica, $5 \%$ em ombro e cotovelo e $4 \%$ em fixadores externos.

Em relação à região geográfica, $67 \%$ residem no Sudeste, $11 \%$ no Sul, $11 \%$ no Nordeste, $8 \%$ no Centro-oeste e 3\% no Norte.

Parâmetros utilizados para decisão sobre o tratamento: $A$ configuração do traço de fratura foi o principal parâmetro utilizado pelos ortopedistas, com um total de $60 \%$ das respostas. A lesão de partes moles ou de estruturas neurovasculares foi citada por $58 \%$; lesões associadas como outras fraturas ou lesões de órgãos vitais, por $34 \%$, e a idade foi citada por $11 \%$.

Classificação utilizada: A grande maioria dos ortopedistas brasileiros (91\%) utiliza alguma classificação para as fraturas diafisárias do fêmur. Dentre os que se baseiam em alguma classificação, a mais selecionada foi a do grupo AO-ASIF, com 84\%. A classificação de Winquist foi referida por $16 \%$.

Opções de tratamento para as fraturas transversas e oblíquas curtas no istmo do fêmur (Figura 1): A haste intramedular bloqueada fresada é o método adotado para o tratamento destas fraturas, com preferência de $54 \%$ dos ortopedistas, seguida pela haste intramedular bloqueada não fresada, com 25\%; placa e parafusos com técnica aberta (25\%); haste intramedular sem bloqueio (18\%); placa ponte (12\%); haste intramedular retrógrada (6\%); tração ou gesso (4\%); fixador externo uniplanar (4\%); llizarov (2\%) e placa onda (1\%).

Opções de tratamento para as fraturas oblíquas longas e cominuídas (Figura 2): A placa ponte foi o método de escolha para 53\% dos ortopedistas, seguida pela haste intramedular bloqueada fresada (37\%); haste intramedular bloqueada não fresada (33\%); placa e parafusos com técnica aberta (15\%); fixador externo uniplanar ( 5\%); llizarov (5\%); haste intramedular retrógrada (4\%); haste intramedular sem bloqueio (3\%); placa onda (3\%) e tração ou gesso (0\%).

Utilização de mesa de tração: Em relação ao uso de mesa ortopédica de tração para a realização de osteossíntese intramedular, 50\% dos ortopedistas a utilizam.

Intervalo de tempo entre o trauma e a cirurgia: Neste item, $18 \%$ dos ortopedistas fixam as fraturas em 24 horas ou menos; $37 \%$ levam de 24 a 48 horas para a realização da cirurgia; $23 \%$ demoram 48 a 72 horas; 16\% levam de 72 horas a 7 dias para operá-las e 7\% demoram mais de uma semana para fixar estas fraturas.

Tração pré-operatória: A tração esquelética pré-operatória é realizada por $72 \%$ dos ortopedistas. A tração cutânea é preferida por 18\%, e 10\% não utilizam tração.

Tempo médio de hospitalização:

Figura 2 - Opções de tratamento para as fraturas oblíquas longas e cominuídas. 
tempo escolhida por $46 \%$ dos ortopedistas; $20 \%$ escolheram entre 6 e 7 dias; $15 \%$, entre 7 e 10 dias; $15 \%$, até 3 dias e $4 \%$ escolheram mais de 10 dias.

Uso de antibiótico nas fraturas diafisárias fechadas do fêmur: Em relação ao uso de antibióticos, $29 \%$ dos ortopedistas usam nas primeiras 24 horas de pós-operatório; $27 \%$, nas primeiras 48horas; $13 \%$, nas primeiras 72 horas; $18 \%$ usam por 1 semana; $2 \%$ usam por mais de 7 dias; $7 \%$ usam somente na indução anestésica, e 4\% não fazem antibioticoprofilaxia.

Complicações mais freqüentes: (Figura 3) A infecção de partes moles foi assinalada por $50 \%$ dos ortopedistas como complicação mais freqüente, seguida por pseudartrose com 38\%; trombose venosa profunda com 30\%; tromboembolismo pulmonar com $14 \%$ e osteomielite com $11 \%$.

Métodos para prevenção de fenômenos tromboembólicos nas fraturas diafisárias do fêmur no adulto: Neste item, $77 \%$ dos ortopedistas utilizam heparina de baixo peso molecular; 14\% usam meias elásticas; 7\% usam warfarin; 5\% utilizam ácido acetil salicílico e 3\% usam foot pump.

\section{DISCUSSÃO}

As fraturas diafisárias do fêmur ocorrem, geralmente, por trauma de alta energia e acometem, predominantemente, adultos jovens, homens (55\%), havendo discreta predominância do lado direito (52\%).

O tratamento das fraturas diafisárias do fêmur é emimentemente cirúrgico porque permite a reabilitação precoce do paciente e diminui o risco de complicações sistêmicas. Alguns parâmetros são secundários, mas devem ser analisados para indicação do tratamento das fraturas diafisárias do fêmur no adulto: configuração do traço de fratura; lesões de partes moles e de estruturas neurovasculares; fraturas associadas e idade. Os ortopedistas brasileiros creditaram maior importância (60\%) à configuração do traço de fratura.

Como classificações mais utilizadas, destacam-se a do grupo AO-ASIF e a de Winquist.

A classificação AO é constituída de um sistema de codificação baseado na localização (proximal, médio ou distal), no traço de fratura e no grau de cominuição. São distinguidos vinte e sete tipos no total.

A classificação de Winquist leva em consideração o grau de cominuição e indica o tipo de tratamento.Tipo I ( fratura com traço simples ou com mínima cominuição ); Tipo II ( cominuição de até 50\% da circunferência da diáfise ); Tipo III ( cominuição de 50 a 100\% da diáfise); Tipo IV (cominuição circunferencial da diáfise, sem contato entre os dois fragmentos maiores depois da redução). Em nosso meio, 91\% dos ortopedistas utilizam alguma classificação, sendo a do grupo AO preferida por $84 \%$, o que caracteriza a grande difusão dos conhecimentos da filosofia AO no Brasil.

Dentre os métodos de tratamento das fraturas diafisárias do fêmur estão as hastes intramedulares bloqueadas ou não, fresadas ou não; as placas de compressão com técnica aberta ou em ponte; os fixadores externos uni ou multiplanares; e métodos alternativos como a tração esquelética e a imobilização com gesso.

Atualmente, tem-se dado preferência à fixação biológica (a foco fechado) das fraturas diafisárias do fêmur com as hastes intramedulares. A utilização de bloqueio estático ou dinâmico na haste é assunto controverso. Em fraturas com traço simples e localizadas no istmo, há autores que não preconizam o bloqueio. Porém, alguns estudos clínicos observaram que o bloqueio estático não influenciou na consolidação destas fraturas ${ }^{1}$. Utilizamos o bloqueio da haste, independentemente do traço ou da localização da fratura(4).

Em casos de redução aberta e osteossíntese com haste intramedular com bloqueio estático, alguns autores recomendam a dinamização entre oito e doze semanas de pós-operatório ${ }^{(5)}$.

A fixação intramedular representa um dos maiores avanços do século passado no tratamento das fraturas.

A primeira citação de fixação intramedular é creditada aos conquistadores no século XVI. Eles observaram que os Incas e os Astecas utilizaram pinos de madeira resinosa no canal medular dos ossos longos para tratamento das pseudartroses. Esse relatório foi encontrado num pequeno parágrafo de arquivos espanhóis, mas não fornece detalhes da técnica, do número de pinos empregado ou dos resultados.

Pinos de marfim foram mencionados por König ${ }^{(6)}$, na Alemanha, em 1913.

Pinos de prata foram utilizados por Schöne, da clínica Kiel, na Alemanha, em 1913. Parece que a prata tendia a controlar a contaminação bacteriana ${ }^{(7)}$.

Hey Groves, na Inglaterra, utilizou a técnica de fixação intramedular em fraturas do fêmur causadas por projétil de arma de fogo durante a Primeira Guerra Mundial. Em três pacientes, os pinos usados lembravam de perto os dispositivos atuais. Havia três padrões de haste: um tubo perfurado, uma haste com secção em forma de $X$ e uma haste sólida redonda, de sua preferência, por não ter fendas que pudessem abrigar infecções ${ }^{(8)}$. Todos os pinos falharam e o autor abandonou o método sem experiências adicionais.

A osteossíntese intramedular bem sucedida, como a conhecemos, começou durante a segunda Guerra Mundial com o trabalho de Küntscher em 1940(9).

Grosse \& Kempf propuseram um método que consistia em uma haste resistente introduzida no canal medular fresado e fixada ao osso por um parafuso proximal e dois distais ao foco da fratura. Enfatizaram a recomendação do uso de mesa ortopédica, intensificador de imagem e proteção das mãos do cirurgião contra a radiação(10) $^{(10}$.

Paschoal, apresentou a haste FMRP (Faculdade de Medicina de Ribeirão Preto). Como vantagens em relação às hastes disponíveis no Brasil até então, dispensavam o uso de intensificadores de imagem e tinham menor custo. O bloqueio era feito por dois parafusos na região trocantérica e dois na porção distal do fêmur ${ }^{(11)}$. Estudos biomecânicos comprovaram que a haste FMRP é mais rígida à flexão e à torção que as hastes AO-ASIF (Haste Universal). Seu comportamento mecânico foi superior quando implantada em fêmores humanos in vitro, em relação à haste $\mathrm{AO}-\mathrm{ASIF}^{(12)}$. Alta taxa 
de consolidação foi observada com a aplicação clínica dessa haste $(94,4 \%)$ e poucas complicações foram relatadas ${ }^{(13)}$.

A osteossíntese com haste intramedular retrógrada apresenta algumas vantagens em relação às anterógradas, nas seguintes situações: pacientes obesos; politraumatizados; com fraturas ipsilaterais da pelve, acetábulo, fêmur proximal e tíbia. Parece não haver diferença em relação ao tempo de consolidação, taxa de pseudartrose e dor no joelho pós-operatória²

Há evidência na literatura de que a fresagem do canal medular reduz as taxas de pseudartrose nas fraturas de ossos longos dos membros inferiores ${ }^{3}$. No Brasil, observa-se que as fraturas transversas e oblíquas curtas no istmo são tratadas, preferencialmente (54\%), com hastes intramedulares bloqueadas fresadas, concordando com as evidências atuais da literatura.

Os fixadores externos encontram aplicabilidade principalmente em pacientes politraumatizados e na estabilização inicial de fraturas complexas e de joelhos flutuantes. Neste estudo, observouse um baixo índice de aplicabilidade dos fixadores externos em fraturas diafisárias do fêmur ( $6 \%$ nas fraturas com traço simples e $10 \%$ nas com traço complexo).

A osteossíntese com placas de compressão, permitindo mobilização ativa do membro e consolidação primária por fixação rígida foi introduzida por Danis, (apud Colton, 1996). Este trabalho teve influência direta na criação do grupo suíço AO (Arbeitsgemeinschaft für Osteosynthesefragen) ${ }^{(14)}$. Diversos autores publicaram seus resultados com o uso das placas no tratamento das fraturas diafisárias do fêmur, enfatizando que as complicações aumentam quando o contato cortical não pode ser obtido. A osteossíntese com o princípio de estabilidade absoluta através de placas de compressão ainda é uma realidade no Brasil, correspondendo ao método de preferência de $25 \%$ dos cirurgiões nas fraturas com traço simples e de 15\%, nas fraturas com traço complexo. É provável que isto se deva ao fato de que muitas regiões do país não disponham de radioscopia para a osteossíntese intramedular ou não possuam equipe treinada para a realização da mesma. Trata-se de um método que promove desvitalização dos tecidos e, conseqüentemente, maior índice de infecção, retarde de consolidação e pseudartrose.

O conceito de fixação biológica das fraturas foi introduzido por Krettek, quando popularizou o termo "MIPPO" (minimally invasive percutaneous plate osteosynthesis), que consiste na colocação de placas por meio de incisões proximal e distal ao foco da fratura, bem como a manipulação indireta dos fragmentos fraturados ${ }^{(15)}$.

No tratamento das fraturas multifragmentárias da diáfise do fêmur, tanto as hastes intramedulares bloqueadas como as placas em ponte permitem bons resultados clínicos, com alto índice de consolidação e poucas complicações ${ }^{(4)}$.

Observou-se que 54\% dos ortopedistas brasileiros optam pela osteossíntese com placa ponte no tratamento das fraturas com traço complexo. A literatura atual considera este método como alternativa confiável para a fixação de fraturas diafisárias do fêmur ${ }^{(5)}$, principalmente em locais onde não há acesso aos implantes intramedulares.

Outra questão controversa é a utilização ou não da mesa de tração para a realização da osteossintese intramedular. Stephen et $\mathrm{al}^{(16)}$ observaram que não houve diferença estatisticamente significativa em relação a tempo de cirurgia e qualidade de redução em um total de 87 pacientes randomizados quanto ao uso ou não de mesa de tração.

Durante um longo período, a tração constituiu uma forma de tratamento definitivo das fraturas diafisárias do fêmur. Atualmente, devido ao conceito da estabilização precoce destas fraturas, tanto a tração cutânea (modificada por Buck 1861) quanto a esquelética, cujo método mais consagrado é a tração balanceada de Thomas-Pearson, tornaram-se métodos temporários, até que se consiga a fixação definitiva da fratura.

A grande maioria dos ortopedistas brasileiros (72\%) prefere a tração esquelética pré-operatória. Talvez, porque 63\% deles não realizam a osteossíntese nas primeiras 24 horas de fratura; seja por motivos operacionais ou por acreditarem que o espasmo muscular inicial poderia dificultar a redução da fratura.

Os portadores de fraturas diafisárias do fêmur são, em sua maioria, politraumatizados, e permanecem internados por longo período( média de 26 dias), o que gera um custo elevado ao tratamento. Neste estudo, verificou-se que $61 \%$ dos ortopedistas relataram um tempo médio de internação de até cinco dias. Isto poderia ser explicado porque, no questionário, enfatizou-se a abordagem da fratura diafisária de fêmur isolada.

É consenso que as fraturas diafisárias do fêmur devam ser operadas precocemente (nas primeiras 24 horas) ${ }^{(17)}$, principalmente devido às possíveis complicações pulmonares inerentes às fraturas de ossos longos. Alguns autores, no entanto, observaram que não houve influência no tempo de consolidação e na mobilidade do joelho em pacientes que foram operados tardiamente $(9,1 \text { dias })^{(4)}$. Pode observar-se, neste estudo, que a maioria dos cirurgiões (63\%) não opera os pacientes nas primeiras 24 horas após o trauma, talvez porque grande parte dos ortopedistas trabalhe em serviços públicos onde as dificuldades operacionais são maiores.

A antibioticoprofilaxia é recomendada mesmo em osteossínteses de fraturas fechadas. Pode ser usada em dose única pré-operatória se o antibiótico atinge concentração inibitória mínima por um período de 12 horas. Pode-se, também, utilizar antibióticos com meia vida mais curta, porém, com múltiplas doses que permitam concentração inibitória mínima por um período de doze horas. As cefalosporinas são os antibióticos mais utilizados ${ }^{(18)}$. Neste item, verificou-se que a grande maioria dos ortopedistas não faz antibioticoprofilaxia conforme as recomendações atuais da literatura, o que poderia justificar o alto índice de citação de infecção pelos mesmos (50\% citam infecção de partes moles e $11 \%$, osteomielite). Se compararmos as taxas de citação de infecção dos indivíduos que fazem antibioticoprofilaxia por 24 horas com aqueles que utilizam antibiótico por 1 semana, verificamos que, no primeiro grupo, 31\% relataram infecção de partes moles e $4 \%$, osteomielite, enquanto que, no segundo grupo, $41 \%$ dos ortopedistas $(p=0,658)$ citaram infecção de partes moles como complicação freqüente, e 10\% $\left(p=0,0438^{*}\right)$ citaram osteomielite.

Quanto à utilização de drenos de sucção no pós-operatório, não existe evidência baseada em estudos randomizados que suportem ou refutem o seu uso em cirurgias ortopédicas para fraturas fechadas ${ }^{(19)}$.

Os fenômenos tromboembólicos são complicações freqüentes nas fraturas femorais. O uso de heparinas não fracionadas ou, preferencialmente, as heparinas de baixo peso molecular reduz a incidência de trombose venosa profunda, assim como os foot pumps ${ }^{(20)}$

Porém, não existe evidência de que haja proteção contra tromboembolismo pulmonar ou diminuição da mortalidade com o seu uso ${ }^{(20)}$. A maioria dos ortopedistas brasileiros (77\%) faz profilaxia antitrombótica com heparinas de baixo peso molecular, 
concordando com as evidências atuais da literatura. É importante citarmos que $7 \%$ dos entrevistados realizam a profilaxia antitrombótica com warfarin. Além de não ser o método de primeira escolha nesta situação, o uso desta droga exige controle periódico com RNI.

Quanto ao tamanho da amostra, conclui-se que a mesma é representativa (507 questionários respondidos integralmente, com total de 518), uma vez que a Sociedade Brasileira de Ortopedia e Traumatologia (SBOT) é composta por 7260 membros, sendo 6581 titulares e 679 associados. Os residentes pertencentes aos serviços credenciados pela SBOT somam um total de 1311 indivíduos.

Comparando o tamanho da amostra deste estudo com o observado na literatura internacional, obteve-se um número significativamente superior ao conseguido por Khalily et al (21), que obtiveram 178 e Bhandari et al(22), com 444 questionários respondidos em estudos transversais sobre fraturas fechadas e fraturas em geral (fechadas e expostas) da diáfise da tíbia, respectivamente.

Em relação à distribuição dos membros em seus respectivos estados, a SBOT concentra 61,39\% dos ortopedistas na região sudeste, $16,76 \%$ na região sul, 7,10\% no Centro-oeste, $12,03 \%$ no Nordeste e 2,69\% no Norte, o que demonstra a representatividade desta amostra, cuja distribuição dos entrevistados é semelhante.

Neste trabalho, 67\% dos questionários foram respondidos por ortopedistas da região sudeste; $11 \%$ do Sul; $11 \%$ do Nordeste; 8\% do Centro-Oeste e 3\% do Norte.

É importante observar as respostas dos ortopedistas em relação às diversas regiões do País, para que se possa verificar se há influência do poder econômico de alguns centros em relação a outros menos favorecidos no tratamento dos pacientes portadores de fraturas diafisárias do fêmur, ou se há diferença de conhecimento técnico sobre a matéria nas diferentes regiões do País.

Neste estudo, não houve diferença estatisticamente significativa em relação aos métodos de fixação dessas fraturas.

\section{REFERÊNCIAS BIBLIOGRÁFICAS}

1. Brumback RJ, Ellison TS, Poka A, Bathon GH, Burgess AR. Intramedullary nailing of femoral shaft fractures. Part III: Long term effects of static interlocking fixation. J Bone Joint Surg Am. 1992; 74:106-12.

2. Ostrum RF, Agarwal A, Lakatos R, Poka A. Prospective comparison of retrograde and anterograde femoral intramedullary nailing. J Orthop Trauma. 2000;14:496-501.

3. Bhandari M, Guyatt GH, Tong D, Adili A, Shaughnessy SG. Reamed versus nonreamed intramedullary nailing of lower extremity long bone fractures: a systematic overview and meta-analysis. J OrthopTrauma. 2000; 14:2-10.

4. Fernandes HJ. Placa em ponte e haste intramedular bloqueada: estudo comparativo no tratamento de fraturas multifragmentárias da diáfise do fêmur [tese]. São Paulo: Escola Paulista de Medicina, Universidade Federal de São Paulo; 2000.

5. Basumallick MN, Bandopadhyay A. Effect of dynamisation in open interlocking nailing of femoral fractures. A prospective randomizaed comparative study of 50 cases with a 2-year follow-up. Acta Orthop Belg. 2002;68:42-7.

6. König F. Ueber die implantation von elfenbein zum erzatz von knochen und gelenkenden. nach experimentellen und klinischen beobachtungen. Beitr Klin Chir. 1913; 85:91.

7. Schöne G. Behandlung fur vorderarmfrakturen mit bolzung. Münch Med Wochenschr. 1913; 60:2327.

8. Groves EW. Ununited fractures with special reference to gunshot injuries and the use of bone grafting. Br J Surg. 1918; 6:203.

9. Küntscher G. Die marknagelung von knochenbrüchen. Arch Klin Chir. 1940; 200:443.

10. Kempf I, Grosse A, Laffourgue D. L'apport du verronillage dans i'enclonage centro-médullaire des os long. Rev Chir Orthop. 1978; 64:635-51.

11. Paschoal FM. Haste bloqueante antitelescopável [dissertação]. Ribeirão Preto: Faculdade de Medicina de Ribeirão Preto, Universidade de São Paulo;1991.

12. Paccola CA, Krettek C, Schandelmeier P, Mannss J. Comparação das propriedades mecânicas das hastes femorais bloqueadas AO-ASIF e FMRP (2a parte). Rev Bras Ortop. 1995; 31:869-77.
A identificação dos indivíduos que responderam aos questionários quanto à sua titularidade também é importante para que se possa verificar se há diferença de resposta estatisticamente significativa entre membros titulares, associados e residentes de ortopedia. Não se abordou a questão da recertificação, uma vez que só pode se recertificar aquele indivíduo que tem cinco anos ou mais de título de especialista, o que poderia gerar uma falsa idéia de desatualização dos membros da SBOT.

Houve diferença estatisticamente significativa entre as respostas dos indivíduos (quanto à sua titularidade) em relação ao método de tratamento das fraturas com traço simples e complexo. Dentre os residentes de Ortopedia, $45 \%\left(p=0,000^{\star}\right)$ realizam a osteossíntese com haste intramedular bloqueada fresada, considerada o método de escolha para as fraturas diafisárias do fêmur. O mesmo método é utilizado por 34\% dos membros titulares e $24 \%$ dos associados à SBOT. Nas fraturas com traço complexo, 30\% ( $\left.p=0,000^{\star}\right)$ dos residentes utilizam este método, comparado com $24 \%$ dos titulares e $11 \%$ dos associados.

Optou-se pela não identificação dos autores no título do questionário, pois conforme observação de Bhandari(23) em 2003, nos Estados Unidos, a colocação de um remetente composto por cirurgiões de instituição conhecida pode influenciar negativamente a taxa de resposta dos indivíduos nos questionários.

\section{CONCLUSÕES}

Conclui-se que, referente ao tratamento das fraturas diafisárias fechadas do fêmur no adulto, a maioria dos ortopedistas brasileiros: considera o traço da fratura como parâmetro principal para decisão do tratamento; utiliza a classificação AO-ASIF; realiza osteossíntese com haste intramedular bloqueada fresada para as fraturas transversas e oblíquas curtas no istmo e com placa ponte nas fraturas com traço complexo; utiliza tração esquelética pré-operatória; tem a infecção como complicação mais freqüente e realiza a profilaxia antitrombótica com heparina de baixo peso molecular.

13. Fernandes HJ, Reis FB, Köberle G, Fallopa F, Christian RW. Tratamento das fraturas diafisárias e instáveis do fêmur com haste intramedular bloqueada. Rev Bras Ortop. 1997; 32:418-24.

14. Colton CL. The history of fracture treatment. In: Browner BD, Jupiter JB, Levine AM, Trafton PG. Skeletal trauma. Philadelphia: Saunders; 1996. p.14-18.

15. Krettek C, Schandelmaier P, Miclau T. Minimally invasive percutaneous plate osteosynthesis (MIPPO) using the DCS in proximal and distal femoral fractures. Injury. 1997; 28:20-30.

16. Stephen DJ, Kreder HJ, Schemitsch EH, Conlan LB, Wild L, McKee MD. Femoral intramedullary nailing: comparison of fracture-table and manual traction. A prospective, randomized study. J Bone Joint Surg Am. 2002;84:1514-21.

17. Lawrence BB, Kenneth DJ, Weigelt J, Sheinberg R. Early versus delayed stabilization of femoral fractures. J Bone Joint Surg Am. 1989; 71:336-40.

18. Gillespie WJ, Walenkamp G. Antibiotic prophylaxix for surgery for proximal femoral and other closed long bone fractures. Cochrane Database Syst Rev. 2001;(1):CD000244.

19. Parker MJ, Roberts C. Closed suction surgical wound drainage after orthopaedic surgery. Cochrane Database Syst Rev. 2001; (4):CD001825.

20. Handall HH, Farrar MJ, Mc Birnie J, Tytherleigh-Strong G, Milne AA, Gillespie WJ. Heparin, low molecular weight heparin and physical methods for preventing deep vein thrombosis and pulmonary embolism following surgery for hip fractures. Cochrane Database Syst Rev. 2002;(4):CD000305.

21. Khalily C, Behnke S, Seligson D. Treatment of closed tibia shaft fractures: a survey from the 1997 orthopaedic trauma Association and Ostheosyntesis International Gerard Küntscher Kreis Meeting. J Orthop Trauma. 2000; 14: 577-81.

22. Bhandari M, Guyatt GH, Swiontkowski MF, Tornetta P 3rd, Hanson B, Weaver B et al. Surgeon's preferences for the operative treatment of fractures of the tibial shaft: an international survey. J Bone Joint Surg Am. 2001;83: 1746-52.

23. Bhandari M, Devereaux PJ, Swiontkwski MF, Schemitsch EH, Shankardass K, Sprague S, Guyatt GH. A randomizaded trial of opinion leader endorsement in a survey of orthopaedic surgeons: effect on primary response rates. Int J Epidemiol. 2003; 32:637-63. 